Old Dominion University

ODU Digital Commons

Applied Research Center Publications

Applied Research Center

2013

\title{
Brust-Schiffrin Synthesis of Catalytic Bipodal PdPt Nanoparticles with Some Mechanistic Insights
}

Sangbum Han

Old Dominion University

Vara P. Sheela

Old Dominion University

Wei Cao

Old Dominion University,wcao@odu.edu

Balasubramanian Ramjee

Old Dominion University, bramjee@odu.edu

Follow this and additional works at: https://digitalcommons.odu.edu/arc_pubs

Part of the Inorganic Chemistry Commons, Nanotechnology Fabrication Commons, and the Physical Chemistry Commons

\section{Repository Citation}

Han, Sangbum; Sheela, Vara P.; Cao, Wei; and Ramjee, Balasubramanian, "Brust-Schiffrin Synthesis of Catalytic Bipodal PdPt Nanoparticles with Some Mechanistic Insights" (2013). Applied Research Center Publications. 5.

https://digitalcommons.odu.edu/arc_pubs/5

\section{Original Publication Citation}

Han, S., Sheela, V. P., Cao, W., \& Balasubramanian, R. (2013). Brust-schiffrin synthesis of catalytic bipodal pdpt nanoparticles with some mechanistic insights. RSC Advances, 3(22), 8551-8558. doi:10.1039/c3ra41078a

This Article is brought to you for free and open access by the Applied Research Center at ODU Digital Commons. It has been accepted for inclusion in Applied Research Center Publications by an authorized administrator of ODU Digital Commons. For more information, please contact digitalcommons@odu.edu. 
Cite this: RSC Advances, 2013, 3, 8551

Received 5th March 2013, Accepted 18th April 2013

DOI: $10.1039 / c 3 r a 41078 a$

www.rsc.org/advances

\section{Brust-Schiffrin synthesis of catalytic bipodal PdPt nanoparticles with some mechanistic insights $\dagger$}

\author{
Sangbum Han, ${ }^{a}$ Vara Prasad Sheela, ${ }^{a}$ Wei $\mathrm{Cao}^{\mathrm{b}}$ and Ramjee Balasubramanian*a
}

\section{Introduction}

Bimetallic nanoparticles are attractive candidates in nanoscience and nanotechnology as their physical and chemical properties and ensuing applications can be modulated by varying their size, shape, and composition. ${ }^{1,2}$ Among various bimetallic nanoparticles, PdPt has attracted enormous attention as they can catalyze a wide variety of reactions from hydrogenation to electrooxidation with high $\mathrm{CO}$ and $\mathrm{S}$ tolerance. $^{3-5}$ The solution phase synthesis of anisotropic nanostructures with face centered cubic metals such as Pt and Pd is extremely challenging, as they have no intrinsic propensity to form anisotropic structures. ${ }^{6}$ However, recent synthetic advances have resulted in the fabrication of controlled PdPt bimetallic nanostructures such as tetrahedrons, ${ }^{7}$ nanocubes, ${ }^{7,8}$ star shaped decahedrons, ${ }^{9}$ nanodendrites, ${ }^{10,11}$ nanoplates, ${ }^{12}$ hollow nanocubes ${ }^{13}$ and core-shell architectures. ${ }^{14}$ Typically these approaches involve high temperature reduction of metals salts ${ }^{7,8}$ or decomposition of organometallic precursors. ${ }^{13}$ Often strategies for shape control rely on structure directing agents which restrict the growth of nanoparticles in certain directions by preferentially adsorbing to select crystallographic planes of the growing nanoparticles. ${ }^{15,16}$ Despite the recent advances the methods for shape control are far less developed when compared to size control. ${ }^{15}$ The specific details of how surfactant ligands influence the

\footnotetext{
${ }^{a}$ Department of Chemistry and Biochemistry, 4541 Hampton Blvd., Old Dominion University, Norfolk, VA, USA. E-mail: bramjee@odu.edu; Tel: +1 757-683-3039 ${ }^{b}$ Applied Research Center, Old Dominion University, Newport News, VA, USA $\dagger$ Electronic supplementary information (ESI) available: UV-vis spectra, histogram of anisotropic nanostructures, STEM and TEM micrographs, EDS analysis and NMR data. See DOI: 10.1039/c3ra41078a
}

shape selection of nanoparticles are not completely understood. ${ }^{6}$

Over the years, Brust-Schiffrin two-phase reduction has played a prominent role in the synthesis of metal nanoparticles. ${ }^{17}$ Initially, it was used for the formation of thiolate protected gold nanoparticles, but has currently been extended to other ligands ${ }^{18}$ and the formation of various mono and bimetallic nanoparticles. ${ }^{19}$ Until recently, ${ }^{20}$ the prevalent theory was that formation of the metal-thiolate polymers preceded the reduction of the metal salt. Current understanding is that a lag time exists between the formation of the nanoparticle and the binding of surfactant to it. ${ }^{20,21}$ Tong and coworkers have recently suggested that the synthesis of the nanoparticles in Brust-Schiffrin method occurs inside an inverse micelle nanoreactor formed by tetraoctylammonium bromide (TOABr). ${ }^{21}$ They proposed that the chalcogenide surfactant ligands present in the organic medium (outside the inverse micelle) control the size of the nanoparticles. ${ }^{21}$ In this article, we show that the resorcinarene amine surfactant (Fig. 1) plays a much larger role in that it is part of the inverse micelle and can profoundly influence the shape and composition of the bimetallic nanoparticles formed.

The reduction of Pt and Pd precursors in the presence of a multidentate resorcinarene amine surfactant (Fig. 1) under Brust-Schiffrin conditions directly leads to the formation of bipodal, Pt rich, crystalline PdPt bimetallic nanoparticles. Resorcinarene amine ${ }^{22}$ combines the benefits of both a multidentate surfactant and an amine ligand. Multidentate ligands derived from macrocyclic building blocks such as resorcinarene $^{23,24}$ and cyclodextrin ${ }^{25}$ functionalized with hydroxyl, ${ }^{23}$ thiol $^{25}$ and amidoamine ${ }^{24}$ headgroups have been used for the synthesis of almost spherical metal nanoparticles. Amine based surfactants have been known to form anisotropic 


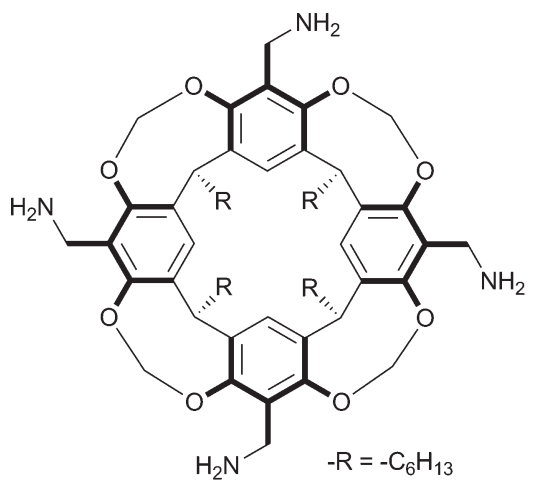

Fig. 1 Resorcinarene amine surfactant.

nanoparticles. ${ }^{26,27}$ However, linear amine stabilized nanoparticles are rarely stable and tend to aggregate into superstructures. $^{28}$ Here we report that the resorcinarene amine stabilized bipodal PdPt nanoparticle dispersions are stable for several months, and can be further subjected to precipitationredispersion cycles without irreversible aggregation.

\section{Experimental}

\section{General remarks}

Hexachloroplatinic acid (Acros Organics, 99.9\%), palladium chloride (Alfa Aesar, 99.9\%), hexylamine (Alfa Aesar, 99\%), tetraoctylammonium bromide (Alfa Aesar, 98+\%), and sodium borohydride (Sigma Aldrich, 98+\%) were used as received. The synthesis of resorcinarene amine surfactant has been described elsewhere. ${ }^{22}$ All organic solvents used in this study were dried and distilled prior to their use. Barnstead Nanopure water $(18.2 \mathrm{M} \Omega \mathrm{cm})$ was used for all syntheses. All glassware used in this study was silanized. Unless otherwise mentioned, all nanoparticle syntheses described in this article were carried out at $0{ }^{\circ} \mathrm{C}$.

\section{Bimetallic nanoparticles by Brust-Schiffrin synthesis}

To a solution of $\mathrm{H}_{2} \mathrm{PtCl}_{6}(9.75 \mathrm{mg}, 0.019 \mathrm{mmol})$ and $\mathrm{H}_{2} \mathrm{PdCl}_{4}$ [prepared by mixing $\mathrm{PdCl}_{2}(3.5 \mathrm{mg}, 0.020 \mathrm{mmol})$ with $\mathrm{HCl}(230$ $\mu \mathrm{L}$ of $0.2 \mathrm{~N}$ ) overnight] in water $(5 \mathrm{~mL})$, tetraoctylammonium bromide (31.1 $\mathrm{mg}, 0.057 \mathrm{mmol})$ in chloroform $(10 \mathrm{~mL})$ was added, and vigorously stirred for $30 \mathrm{~min}$ at room temperature under argon atmosphere. During stirring, the color of the aqueous phase changed from yellow to colorless, while that of the organic phase changed from colorless to reddish brown. The organic phase was separated and stirred with a solution of resorcinarene amine $(6.2 \mathrm{mg}, 0.006 \mathrm{mmol})$ in chloroform (10 $\mathrm{mL}$ ) for $30 \mathrm{~min}$ at room temperature. The reaction mixture was subsequently cooled to $0{ }^{\circ} \mathrm{C}$ and a solution of sodium borohydride $(14.5 \mathrm{mg}, 0.383 \mathrm{mmol})$ in ice cold water $(2 \mathrm{~mL})$ was added under argon atmosphere. After $2 \mathrm{~h}$ of stirring at 0 ${ }^{\circ} \mathrm{C}$, the organic layer was collected and washed with copious amounts of water $(20 \mathrm{~mL} \times 3)$. The nanoparticles were purified by a precipitation/redispersion cycle. For precipitation, nanoparticle dispersions in chloroform were mixed with isopropyl alcohol in a $1: 2$ volume ratio and centrifuged at $10000 \mathrm{rpm}$ for $20 \mathrm{~min}$. The precipitate could be readily redispersed in chloroform by gentle shaking.

\section{Monometallic nanoparticles by Brust-Schiffrin synthesis}

Monometallic Pd or Pt nanoparticles were prepared by a procedure similar to that employed for bimetallic nanoparticle synthesis. The concentration of $\mathrm{H}_{2} \mathrm{PtCl}_{6}$ or $\mathrm{H}_{2} \mathrm{PdCl}_{4}$ was kept at $\sim 0.038-0.039 \mathrm{mmol}$ with all other conditions being identical.

\section{Bimetallic nanoparticles with hexylamine surfactant}

The synthesis of PdPt bimetallic nanoparticles with hexylamine surfactant was very similar to that described with resorcinarene amine surfactant except that the concentration of hexylamine was kept at $0.025 \mathrm{mmol}$.

\section{Bimetallic nanoparticles by a reversed Brust-Schiffrin synthesis}

This synthesis is different from the conventional BrustSchiffrin synthesis described above in that resorcinarene amine in chloroform $(2 \mathrm{~mL})$ was added to the reaction mixture either $10 \mathrm{~s}$ or $5 \mathrm{~min}$ after the addition of sodium borohydride in water. Note that the total amounts of the reagents and solvents were kept constant in both reversed and conventional Brust-Schiffrin synthesis.

\section{Bimetallic nanoparticles by a monophasic approach}

A solution of $\mathrm{H}_{2} \mathrm{PtCl}_{6}(9.75 \mathrm{mg}, 0.019 \mathrm{mmol})$ and $\mathrm{H}_{2} \mathrm{PdCl}_{4}$ (prepared from $3.34 \mathrm{mg}$ of $\left.\mathrm{PdCl}_{2}, 0.019 \mathrm{mmol}\right)$ in water $(5 \mathrm{~mL})$ was phase transferred from aqueous phase into chloroform $(10 \mathrm{~mL})$ with the aid of TOABr $(31.1 \mathrm{mg}, 0.057 \mathrm{mmol})$. The organic phase was separated, evaporated, and subsequently dried under vacuum for $\sim 2$ days. The TOA stabilized metal salts were dissolved in chloroform $(10 \mathrm{~mL})$ and a solution of resorcinarene amine $(6.3 \mathrm{mg}, 0.006 \mathrm{mmol})$ in chloroform (5 $\mathrm{mL}$ ) was added to it and stirred at room temperature for 30 min. The reaction mixture was cooled to $0{ }^{\circ} \mathrm{C}$ and tetrabutylammonium borohydride (96.9 $\mathrm{mg}, 0.377 \mathrm{mmol}$ ) in chloroform $(7 \mathrm{~mL})$ was added under argon atmosphere and almost immediately the reaction mixture turned black. After stirring for $2 \mathrm{~h}$ at $0{ }^{\circ} \mathrm{C}$, the reaction mixture was subsequently washed with water $(20 \mathrm{~mL} \times 3)$.

\section{Suzuki cross-coupling reaction}

To a dispersion of phenyl boronic acid (18.2 $\mathrm{mg}, 0.149 \mathrm{mmol})$, iodobenzene $(0.01 \mathrm{~mL}, 0.098 \mathrm{mmol})$, and $\mathrm{K}_{2} \mathrm{CO}_{3}(27.2 \mathrm{mg}$, $0.197 \mathrm{mmol})$ in toluene $(9 \mathrm{~mL})$, resorcinarene amine stabilized (PdPt, Pt or Pd) metal nanoparticles ( $0.6 \mathrm{mg}$ metal content) in chloroform $(3 \mathrm{~mL})$ were added and refluxed for 2 days. The product formation was analyzed by HPLC analysis.

\section{DLS sample preparation}

To a solution of TOABr in chloroform $(6.2 \mathrm{mg}, 0.011 \mathrm{mmol}, 2$ $\mathrm{mL})$, water $(1 \mathrm{~mL})$ was added and stirred at room temperature for $30 \mathrm{~min}$. The organic layer was separated and either a resorcinarene amine solution in chloroform $(1.3 \mathrm{mg}, 0.0013$ mmol, $2 \mathrm{~mL}$ ) or chloroform ( $2 \mathrm{~mL}$ ) was added, stirred at room temperature for $30 \mathrm{~min}$, and used as such for DLS measurements. 


\section{Characterization}

TEM and EDS analysis were carried out using a JEOL JEM$2100 \mathrm{~F}$ field emission microscope operating at $200 \mathrm{kV}$ equipped with an Oxford INCAx-sight EDS detector and a Gatan SC1000 ORIUS CCD camera (11 megapixel). Unless otherwise mentioned, all EDS analysis reported in this manuscript were carried out in TEM mode. TEM samples were prepared by dropcasting chloroform dispersions of nanoparticles on carbon film coated copper grids. Nanostructure dimensions and $d$ spacings were measured using Image J software. Unless otherwise mentioned as as-prepared samples, all TEM images were obtained from precipitated and redispersed (purified) nanoparticles. UV-vis spectra were recorded on a Cary-5000 UV-Vis-NIR spectrophotometer. DLS analysis was carried out at $25{ }^{\circ} \mathrm{C}$ in a Malvern Zetasizer ZEN 3200 model. ${ }^{1} \mathrm{H}$-NMR spectra were recorded on a Varian Oxford $400 \mathrm{MHz}$ instrument. HPLC analysis of catalytic reactions was carried out in a Thermoelectron spectrum HPLC equipped with a PDA detector, and C18 functionalized Hypersil gold column (250 $\times 4.6 \mathrm{~mm}$ dimension, $5 \mu \mathrm{m}$ particle size) using $60 \% \mathrm{CH}_{3} \mathrm{CN} /$ $\mathrm{H}_{2} \mathrm{O}\left(1 \mathrm{~mL} \mathrm{~min}^{-1}\right)$ as the mobile phase.

\section{Results and discussion}

\section{V-shaped PdPt bimetallic nanoparticles and factors influencing their formation}

We employed a modified Brust-Schiffrin procedure ${ }^{29}$ for the synthesis of PdPt bimetallic nanoparticles in the presence of resorcinarene amine surfactant. Briefly, tetraoctylammonium stabilized Pd and Pt salts in chloroform were reduced with aqueous sodium borohydride solution in the presence of resorcinarene amine surfactant at $0{ }^{\circ} \mathrm{C}$ for $2 \mathrm{~h}$. The as-prepared nanoparticles obtained were purified by a precipitationredispersion approach. The UV-vis spectra of the PdPt bimetallic nanoparticles showed an almost featureless spectrum except for a shoulder around $280 \mathrm{~nm}$ (Fig. S1, ESI $\dagger$ ). This is consistent with the fact that the surface plasmon resonances of the constituent metals, Pd and Pt, are usually in the UV region. ${ }^{30}$ Resorcinarene amine stabilized nanoparticle dispersions were stable even after months of storage.

Transmission electron microscopic (TEM) analysis of the purified product (Fig. 2a) revealed the formation of anisotropic nanostructures along with some spherical nanoparticles. ${ }^{31}$ The formation of anisotropic structures is noteworthy as Brust-Schiffrin reaction routinely yields nominally spherical nanoparticles with $<6 \mathrm{~nm}$ diameter. ${ }^{17,32}$ A closer look at the anisotropic structures (Fig. 2b and S2, ESI $\dagger$ ) showed that $>$ $80 \%$ of them (from $n>250$ particles) had a V-shape or a variant on the $\mathrm{V}$-shape such as one-arm bent $\mathrm{V}$, both arms bent $\mathrm{V}$, wide-open $\mathrm{V}$ and $\mathrm{U}$ shaped nanoparticles (Fig. S3, ESI $\dagger$ ). When compared to as-prepared nanoparticles (Fig. S4, ESI $\dagger$ ) the purified samples were noticeably richer in V-shaped and one-arm bent V-shaped nanoparticles (Fig. S3, ESI $\dagger$ ). Such V-shaped nanostructures and its variants are collectively referred to as bipodal nanostructures in this manuscript. The dominant formation of bipodal nanostructures is notable as typical solution based synthesis of branched nanocrystals

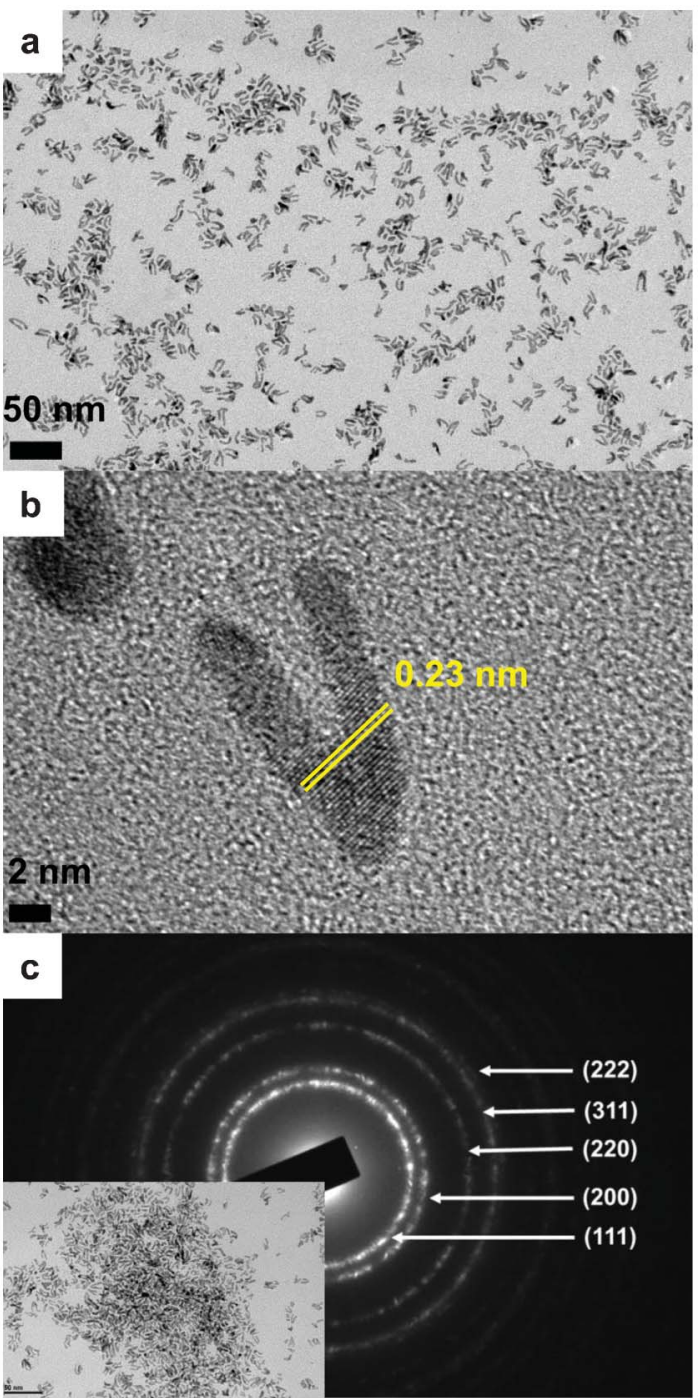

d

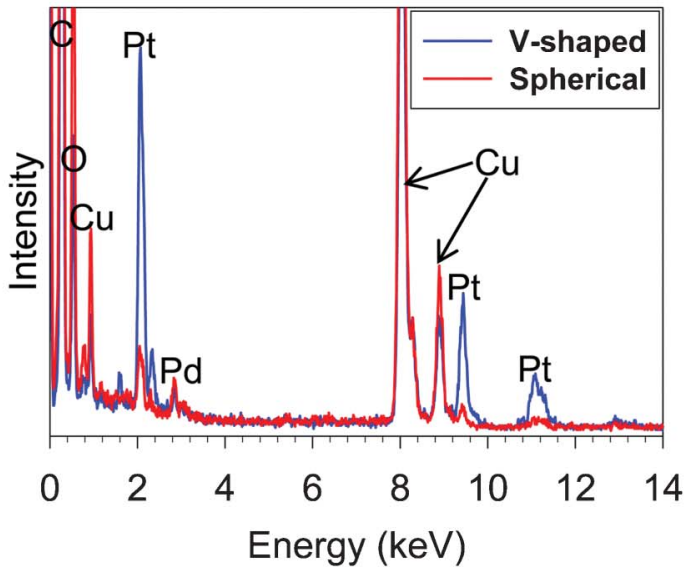

Fig. 2 TEM (a-b) and SAED ( $c$, of the region shown in the inset) of PdPt bimetallic nanoparticles prepared in the presence of resorcinarene amine surfactant by Brust-Schiffrin synthesis at $0{ }^{\circ} \mathrm{C}$. (d) EDS analysis of $\mathrm{V}$-shaped (blue trace) and spherical (red trace) nanoparticles in as-prepared samples. 
tends to produce mixtures of multipods. ${ }^{33}$ Often the length of both arms of the V-shaped nanoparticles was not equal. The lengths of the longer and shorter arms were $10.0 \pm 2.7 \mathrm{~nm}$ and $8.4 \pm 2.4 \mathrm{~nm}$ respectively, and the ratio of longer to shorter arm lengths in individual nanoparticles was $1.2 \pm 0.15$ $(\mathrm{n}=75)$. The cross-sectional diameter of the arms was $2.6 \pm$ $0.4 \mathrm{~nm}$. The HRTEM image (Fig. 2b) of these crystalline nanoparticles showed a typical $d$-spacing of $0.23 \mathrm{~nm}$, consistent with Pt and Pd (111) planes. The selected area electron diffraction (SAED) of a collection of V-shaped structures (inset) showed continuous rings that could be indexed to Pt or Pd (111), (200), (220), (311) and (222) planes (Fig. 2c).

We employed single particle energy dispersive spectroscopy (EDS) to measure the Pt and Pd content in these nanoparticles (Fig. 2d). In addition to these, Cu originating from the grid was also observed. ${ }^{34}$ The V-shaped nanoparticle showed a $\mathrm{Pt} / \mathrm{Pd}$ ratio of 7.6 (Fig. 2d, blue trace), substantially different from the $1: 1$ ratio of Pt and Pd precursors employed in the BrustSchiffrin synthesis. This higher Pt content was widely prevalent, as single particle EDS of 8 different particles from 4 different preparations (including 2 purified samples) gave a $\mathrm{Pt} / \mathrm{Pd}$ ratio of $8.7 \pm 2.9$. Note that there were no significant differences in the $\mathrm{Pt} / \mathrm{Pd}$ ratio of the anisotropic nanoparticles in as-prepared $(9.9 \pm 2.1)$ and purified $(8.0 \pm 3.3)$ samples. To probe the uniformity of the distribution of Pt and Pd, or lack thereof, EDS analysis was carried out under STEM mode at distinct spots of the anisotropic V-shaped nanoparticles (with a beam spot size of $0.5 \mathrm{~nm}$ ) and their Pt : Pd ratio measured. As shown in Fig. S5, ESI $\dagger$, the Pt : Pd ratios were measured at the center, and at the end of both arms. Consistently a relatively much lower $\mathrm{Pt}$ : Pd ratio was observed at the center when compared to those measured at the ends. Notably, the Pt : Pd ratio on both arms were not identical and in all cases but one the shorter arm had a higher Pt : Pd ratio than the longer arm. Analysis of 5 different anisotropic structures yielded a typical Pt : Pd ratio of $3.9 \pm 0.8$ at the center of the V-shaped nanoparticle, $6.9 \pm 2.3$ at the end of the relatively shorter arm and $5.6 \pm 3.2$ at the end of the relatively longer arm. The spherical nanoparticles formed in the same synthesis had a Pt/Pd ratio of 0.75 (Fig. 2d, red trace). Given the minimal lattice mismatch and the excellent miscibility of $\mathrm{Pt}$ and $\mathrm{Pd}$ in bulk, ${ }^{2,10}$ the spontaneous formation of $\mathrm{Pt}$ rich bipodal nanoparticles with such anisotropic elemental distribution is rather surprising. This result is also noteworthy as the groups of Toshima, ${ }^{35}$ Crooks, ${ }^{36}$ and Sun $^{37}$ have demonstrated that the elemental composition of the PdPt nanoparticles was almost identical to the $\mathrm{Pd}$ and $\mathrm{Pt}$ precursor ratio employed in the synthesis over a range of compositions.

Both bipodal and spherical nanoparticles are formed as early as $5 \mathrm{~min}$ after the addition of sodium borohydride (Fig. S6, ESI $\dagger$ ). The spherical nanoparticles are present even after $2 \mathrm{~h}$ of reaction. Given the small, yet discernible difference between the cross-section of the bipodal PdPt nanostructures $(2.6 \pm 0.4$ $\mathrm{nm})$ and the dimensions of the spherical PdPt nanoparticles $(2.0 \pm 0.4 \mathrm{~nm})$, both may be formed concurrently from homogeneous nucleation events. Even though such a nucleation event is often not invoked for the formation of branched structures, recently Xia and coworkers have shown that both homogeneous and heterogeneous nucleation events lead to the formation of dendritic bimetallic nanostructures. ${ }^{6,10,38}$ Further, we ruled out Pd seed mediated growth of V-shaped PdPt nanoparticles, as the reduction of Pt salts in the presence of dominantly spherical Pd nanoparticle seeds yielded mostly spherical nanoparticles (Fig. S7, ESI $\dagger$ ). Our results are consistent with the observations of Tilley and coworkers, who have shown that amine ligand stabilized Pd nanoparticle seeds retain their morphology during subsequent growth stages. ${ }^{39}$

The elemental composition of the anisotropic nanoparticles could be varied by varying the reaction temperature. Though reduction at room temperature led to the formation of crystalline bipodal nanoparticles (Fig. S8a and S8b, ESI $\dagger$ ), their Pt : Pd ratio was $5.6 \pm 1.1$ (Fig. S8c, ESI $\dagger$ ). The $\mathrm{H}_{2} \mathrm{PtCl}_{6}$ used in synthesis of bimetallic nanoparticles was replaced with $\mathrm{K}_{2} \mathrm{PtCl}_{4}$ to probe the influence of the oxidation state of Pt. This reaction also yielded anisotropic nanoparticles with a V-shape and variants of V-shape along with spherical nanoparticles (Fig. S9, ESI $\dagger$ ). The single particle EDS analysis of 5 different anisotropic nanoparticles gave a $\mathrm{Pt} / \mathrm{Pd}$ ratio of $13.9 \pm 2.6$, a somewhat higher ratio than those obtained with $\mathrm{H}_{2} \mathrm{PtCl}_{6}$. This experiment indicates that irrespective of the oxidation state of Pt (+4 vs. +2$)$ anisotropic Pt rich V-shaped PdPt bimetallic nanoparticles are obtained in the presence of resorcinarene amine surfactant.

To evaluate the generality of bipodal nanoparticle formation, monometallic Pt and Pd nanoparticles were synthesized in the presence of resorcinarene amine under Brust-Schiffrin conditions. When $\mathrm{H}_{2} \mathrm{PtCl}_{6}$ was reduced in the presence of resorcinarene amine, crystalline anisotropic nanostructures including a few vaguely reminiscent of V-shaped nanostructures (Fig. 3a and 3b) were observed. With Pd, the TEM analysis primarily showed the formation of spherical nanoparticles along with a few polycrystalline V-shaped nanostructures (Fig. 3c and 3d). These experiments suggest that the dominant formation of crystalline, bipodal nanoparticles requires the presence of both $\mathrm{Pt}$ and $\mathrm{Pd}$. It is worth noting that the presence of a second metal or foreign ion has been

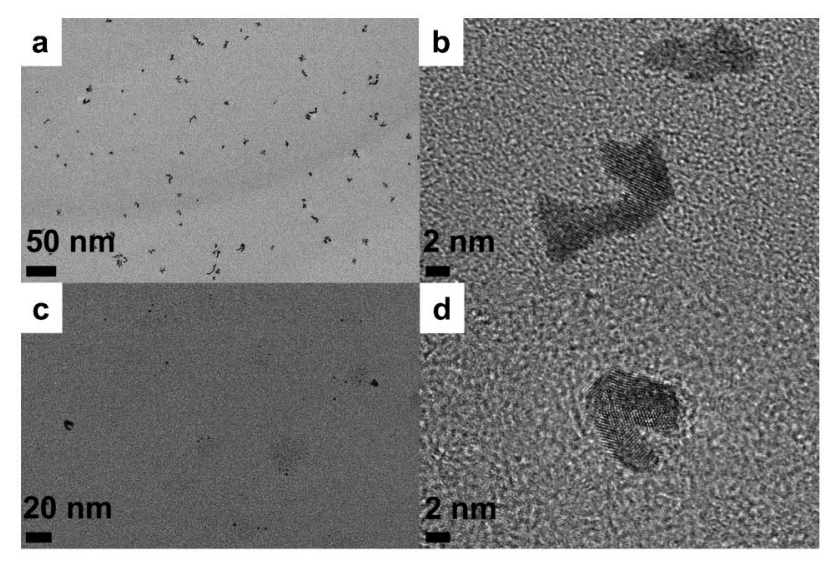

Fig. 3 TEM analysis of monometallic Pt (a-b) and Pd (c-d) nanoparticles prepared in the presence of resorcinarene amine surfactant. 
implicated in the observed shape control of various nanoparticles. $^{40}$

When resorcinarene amine was replaced with hexylamine surfactant, a mixture of spherical and slightly elongated nanoparticles with a Pt: Pd ratio of $\sim 1$ was obtained (Fig. 4). This is not entirely surprising, as the coreduction of Pt and Pd precursors with commonly employed surfactants such as $\mathrm{PVP}^{35}$ or oleylamine ${ }^{37}$ has resulted in the formation of smaller, nominally spherical nanoparticles.

A monophasic synthesis was carried out to probe the importance of inverse micelle in the formation of $\mathrm{V}$-shaped PdPt bimetallic nanoparticles. The TEM analysis of the product showed mostly spherical nanoparticles along with a few elongated structures (Fig. S10, ESI $\dagger$ ). These experiments suggest that the presence of resorcinarene amine alone is not sufficient for the synthesis of V-shaped nanoparticles and that water and hence inverse micelles are also necessary.

The role of resorcinarene amine surfactant in Brust-Schiffrin synthesis

To probe the exact role of the resorcinarene amine surfactant in nanoparticle formation reversed Brust-Schiffrin syntheses ${ }^{21}$ was carried out, where the resorcinarene amine surfactant was added after the addition of aqueous sodium borohydride solution. Two experiments were carried out with a delay time of $10 \mathrm{~s}$ and $5 \mathrm{~min}$ between the additions, and noticeable amount of insoluble material was observed in the latter
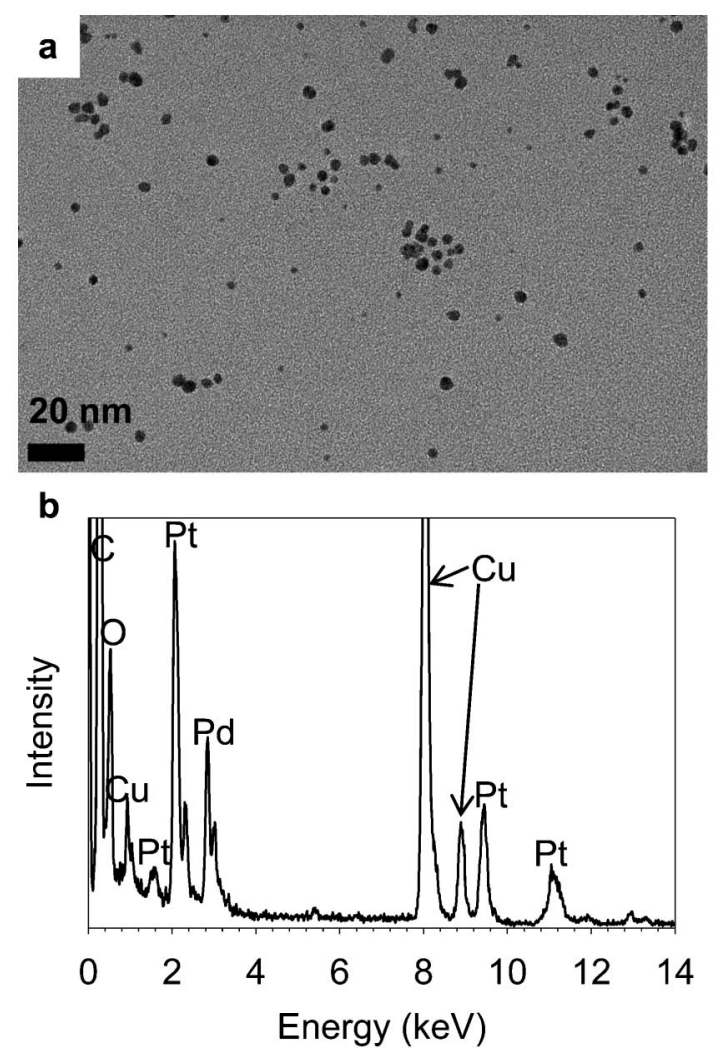

Fig. 4 (a) TEM and (b) EDS of PdPt bimetallic nanoparticles prepared with hexylamine surfactant at $0{ }^{\circ} \mathrm{C}$. synthesis. Significant amounts of V-shaped nanoparticles were obtained in the reversed Brust-Schiffrin synthesis with a $10 \mathrm{~s}$ delay, (Fig. 5a and S11a, ESI $\dagger$ ). However with a 5 min delay, only a few of the anisotropic structures obtained were vaguely reminiscent of V-shaped nanoparticles (Fig. 5b and S11b, ESI $\dagger$ ). The crystallinity of nanoparticles prepared by reversed Brust-Schiffrin synthesis differed substantially with the delay time. While the anisotropic bimetallic nanoparticles formed with a $10 \mathrm{~s}$ delay were crystalline (Fig. S12a, ESI $\dagger$ ), those formed with a $5 \mathrm{~min}$ delay were polycrystalline (Fig. S12b, ESI $\dagger$ ). The single particle EDS measurements of 3 different bipodal nanoparticles (Fig. 5c, blue trace) obtained from the reversed Brust-Schiffrin synthesis with a $10 \mathrm{~s}$ delay showed a Pt : Pd ratio of $5.5 \pm 0.6$. On the other hand with a 5 min
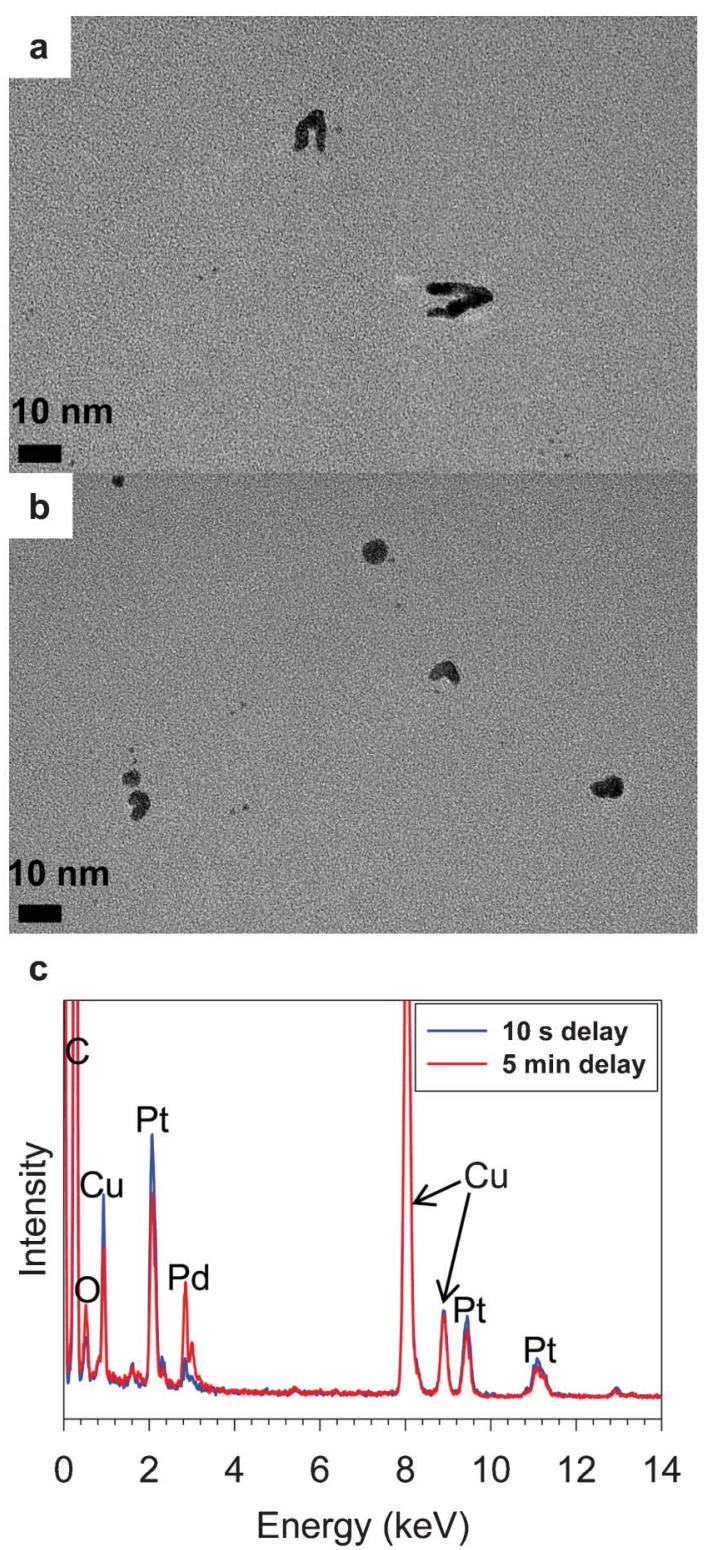

Fig. 5 TEM (a-b) and single (anisotropic) particle EDS (c) analysis of PdPt nanoparticles prepared by reversed Brust-Schiffrin syntheses with a delay time of $10 \mathrm{~s}(\mathrm{a}$, blue trace in $\mathrm{c})$ and $5 \mathrm{~min}$ (b, red trace in $\mathrm{c}$ ). 
delay, the anisotropic structures formed (Fig. $5 \mathrm{c}$, red trace) had a Pt : Pd ratio of $0.9 \pm 0.3$, though occasionally slightly Pd rich anisotropic structures were also obtained. These experiments demonstrate the crucial role played by the resorcinarene amine surfactant in determining the shape, composition and crystallinity of the anisotropic bimetallic nanoparticles formed under Brust-Schiffrin conditions.

The key difference between the reversed and conventional Brust-Schiffrin syntheses is the absence of the resorcinarene amine surfactant in the former during the formation of the nuclei and eventually nanoparticles. We carried out dynamic light scattering (DLS) analysis of the inverse micelles formed with $\mathrm{TOABr}$ and a mixture of $\mathrm{TOABr}$ and resorcinarene amine surfactant. In the case of TOABr based inverse micelles a broad peak was obtained at $\sim 712 \mathrm{~nm}$, whereas TOABr and resorcinarene amine derived inverse micelles gave a sharp peak at $\sim 79 \mathrm{~nm}$ (Fig. 6). On the basis of these DLS studies and the results of the reversed Brust-Schiffrin syntheses, we propose the incorporation of the resorcinarene amine surfactant in the TOABr derived inverse micelles, where the nanoparticles are being formed. Given the lack of solubility of resorcinarene amine (or its hydrochloride salt) in water, we expect it to be on the surface of the inverse micelle with the amine headgroups pointed towards the water pool, rather than being solubilized inside the micelle. Although these results appear to contradict the recent work of Tong and coworkers, ${ }^{21}$ it agrees with Pileni and coworkers ${ }^{27}$ who have invoked the involvement of surfactant along with $\mathrm{TOABr}$ in the inverse micelle formation.

We probed the interaction of the phase-transferred metal salts with the inverse micelles by UV-vis spectroscopy (Fig. 7). The band observed $\sim 259 \mathrm{~nm}$ for the aqueous mixture of $\mathrm{Pt}$ and Pd metal salts was red shifted in the presence of inverse micelles (Fig. 7). The $\lambda_{\max }$ of the metal salts in the presence of inverse micelles containing resorcinarene amine showed a lesser red shift $(\sim 266 \mathrm{~nm})$ when compared to the inverse micelles formed exclusively with $\operatorname{TOABr}(\sim 272 \mathrm{~nm})$. While the former appeared yellow, the latter appeared reddish brown. We infer from this data that the resorcinarene amine present in the inverse micelle interacts with the metal salts even prior

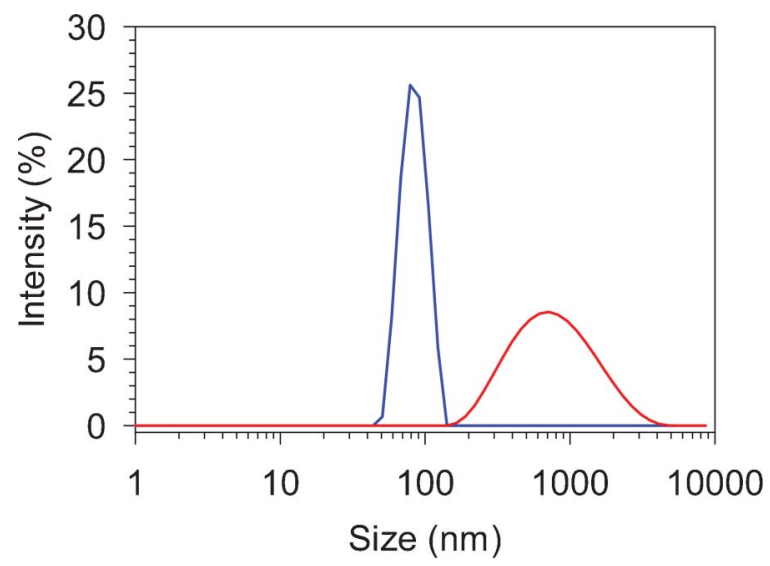

Fig. 6 DLS analysis of inverse micelles formed in chloroform from a) $\mathrm{TOABr}+$ resorcinarene amine (blue trace) and b) TOABr (red trace).

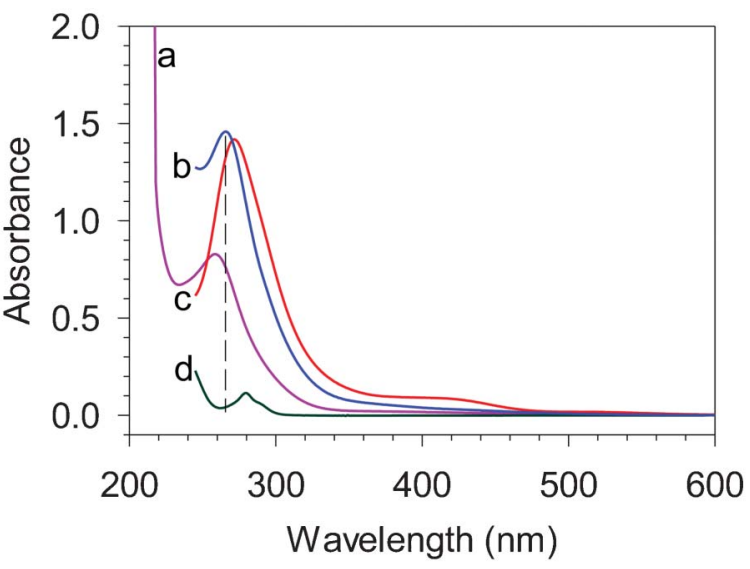

Fig. 7 UV-vis spectra of (a) Pd and Pt salts dissolved in water, (b) inverse micelles formed from $\mathrm{TOABr}$ and resorcinarene amine containing $\mathrm{Pd}$ and Pt salts, (c) inverse micelles formed from $\mathrm{TOABr}$ containing $\mathrm{Pd}$ and Pt salts and (d) resorcinarene amine in chloroform.

to their reduction. Our observation is unlikely to be due to the mere presence of resorcinarene amine, as its UV-vis spectrum showed a more red shifted peak $\left(\lambda_{\max }=280 \mathrm{~nm}\right)$ and much weaker absorbance.

The complexation of metal salts to resorcinarene amine surfactant was further confirmed by ${ }^{1} \mathrm{H}$-NMR spectroscopy (Fig. 8 and S13, ESI $\dagger$ ). The presence of both Pt and Pd led to substantial broadening or shifting of the resorcinarene amine peaks (Fig. S13, ESI $\dagger$ ) such as $\mathrm{O}-\mathrm{CH}_{2}-\mathrm{O}$ protons (5.92 and 4.35 ppm), $\mathrm{CH}$ protons (4.76 ppm) and $\mathrm{N}-\mathrm{CH}_{2}-$ protons (3.6 ppm) (Fig. 8b), while no significant shift in the resorcinarene amine peak positions was observed in the absence of metal salts (Fig. 8a). It is worth noting that the complexation of metal salts by dendrimers has been known to result in the broadening and shift of methylene protons attached to the N. ${ }^{41}$ Further,
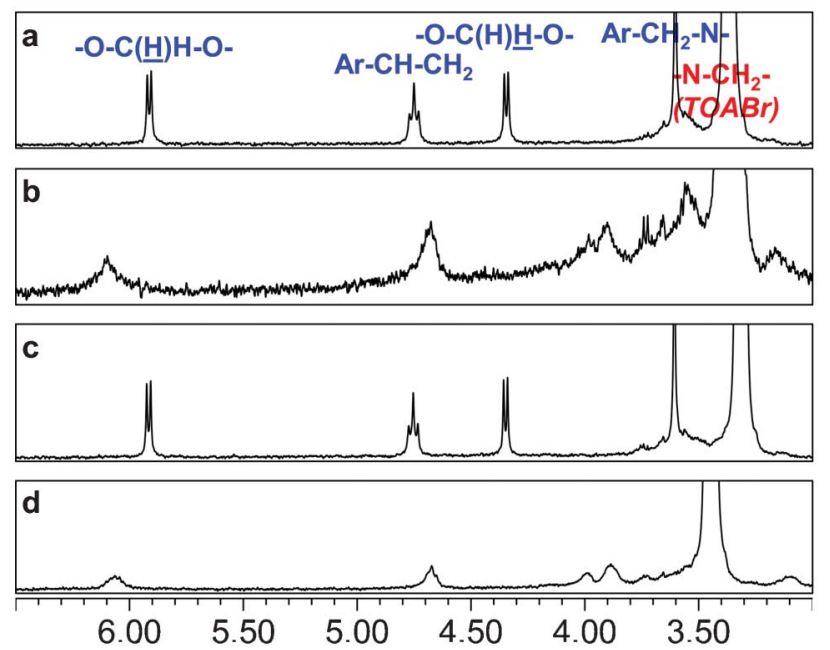

Fig. 8 Partial ${ }^{1} \mathrm{H}$-NMR spectra of inverse micelles formed from TOABr and resorcinarene amine showing the shift of resorcinarene amine peaks (labelled in blue) in the absence of metal salts (a) and presence of both Pd and Pt (b), Pt (c) and $\mathrm{Pd}(\mathrm{d})$ salts. 
NMR studies carried out with Pt or Pd salts showed that the strong complexation of resorcinarene amine was limited to $\mathrm{Pd}$ (Fig. 8d) and not Pt (Fig. 8c). Currently we are investigating the differences in the binding of metal salts to resorcinarene amine surfactant in detail.

Based on these experiments and relevant literature, we believe that the preferential formation of $\mathrm{Pt}$ rich bipodal nanoparticles and Pd rich spherical nanoparticles in BrustSchiffrin synthesis could be due to the differential stabilization of a) Pt and Pd precursors and b) the nuclei formed, both by the resorcinarene amine surfactant. The nucleation stage and in particular the nuclei formed initially have a profound influence on the final shape ${ }^{42}$ and composition of the nanoparticles. Once the nuclei are formed they will be capped with organic surfactants. Studies have shown the binding of amine functional groups to both Pt and Pd metal surfaces ${ }^{43}$ and nanoparticles. ${ }^{27,44}$ However, the stabilization of Pt and Pd metal surfaces by amine surfactant could differ due to potential differences in interaction energies between the ligand and metal surfaces and the adsorption geometries. ${ }^{45}$ It is worth noting that the selective stabilization of Pt metal by amine surfactant has been invoked in the formation of $\mathrm{Au}-\mathrm{Pt}$ bimetallic nanowires. ${ }^{46}$ Detailed mechanistic studies on the influence of surfactant head group in determining the morphology and composition of the bimetallic nanoparticles are currently underway in our laboratory and will be reported in due course.

\section{Catalysis using PdPt bimetallic nanoparticles}

We tested PdPt bipodal nanoparticles as catalysts in Suzuki cross-coupling reaction. ${ }^{47}$ Suzuki reaction between aryl boron derivatives and aryl halides is a versatile and powerful carboncarbon bond forming tool. Though usually accomplished by organopalladium complexes, it has also been catalyzed by a variety of metal nanoparticles. ${ }^{48-50}$ Given the established superiority of $\mathrm{Pd}^{49}$ over $\mathrm{Pt}^{48,51}$ in mediating Suzuki reactions, we chose to evaluate the PdPt bimetallic nanoparticles prepared at $0{ }^{\circ} \mathrm{C}$ as catalysts for the coupling of iodobenzene and phenylboronic acid. ${ }^{52}$ Table 1 summarizes the yields of

Table 1 Resorcinarene amine stabilized bi- and mono- metallic nanoparticles were used as catalysts for Suzuki cross-coupling reaction between iodobenzene and phenylboronic acid. The yield of biphenyl was determined by HPLC analysis

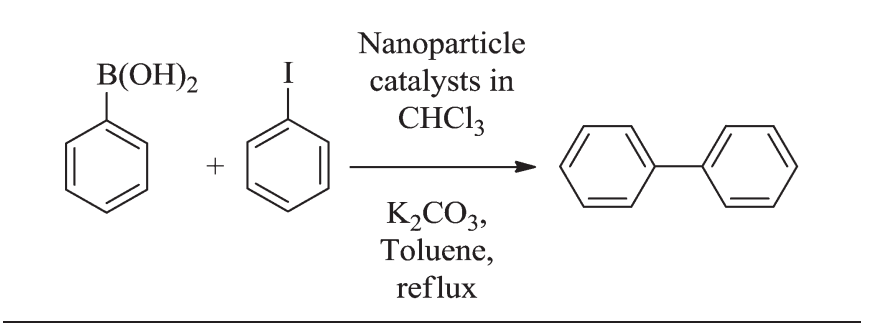

\begin{tabular}{ll}
\hline Nanoparticle catalysts & Yield (\%) \\
\hline PdPt (as prepared) & 72 \\
PdPt (purified) & 49 \\
Pd (as prepared) & 59 \\
Pt (as prepared) & $<2$
\end{tabular}

biphenyl obtained in the presence of bimetallic and monometallic (as control) nanoparticle catalysts.

The as-prepared and purified PdPt bimetallic nanoparticles yielded $72 \%$ and $49 \%$ of biphenyl respectively. Even under these vigorous reaction conditions $\left(>110{ }^{\circ} \mathrm{C}\right.$ for $\left.48 \mathrm{~h}\right)$ the nanoparticle dispersions were visibly stable after the catalysis. TEM analysis of the nanoparticles after catalysis showed that they were intact but aggregated (Fig. S14, ESI†). This observation is remarkable as El-Sayed and coworkers have shown that a majority of tetrahedral Pt nanoparticles undergo a shape change to spherical after just $24 \mathrm{~h}(2 \times 12 \mathrm{~h}$ cycles of catalysis $)$ of reflux under similar reaction conditions. ${ }^{51}$ Surprisingly ${ }^{48}$ the as-prepared bimetallic nanoparticles were more active than the monometallic Pd nanoparticles which gave a 59\% yield of biphenyl, under our reaction conditions. Consistent with literature observations, ${ }^{48}$ the monometallic Pt nanoparticles resulted in $<2 \%$ conversion.

\section{Conclusions}

We have shown that the Brust-Schiffrin reduction of Pt and Pd precursors in the presence of a multidentate resorcinarene amine surfactant leads to the formation of Pt rich, crystalline, PdPt nanoparticles of a V-shape or variants on the V-shape along with some Pd rich PdPt spherical nanoparticles. We have studied the influence of resorcinarene surfactant, metal precursors, temperature, and reaction medium on the formation of bimetallic nanoparticles in detail. Our experiments have unambiguously demonstrated the crucial role played by the resorcinarene amine surfactant and water, and hence inverse micelles, in the formation of bipodal nanoparticles. On the basis of reversed Brust-Schiffrin synthesis and DLS analysis, we propose the incorporation of the resorcinarene amine surfactant in the TOABr derived inverse micelles, where the bimetallic nanoparticles are being formed. Further, we have also shown that the resorcinarene amine complexes with the Pd salts prior to their reduction. Remarkably, we show that the composition of the bipodal PdPt nanoparticles could be varied substantially by varying the order of addition of resorcinarene amine surfactant and sodium borohydride, and the delay time between the additions. The utility of such resorcinarene amine stabilized bipodal PdPt nanoparticles in mediating Suzuki cross-coupling reactions has been demonstrated.

\section{Acknowledgements}

The authors thank Old Dominion University (ODU) for financial support. TEM and EDS analysis were carried out at the Applied Research Center, ODU. The authors thank Professors Jennifer Poutsma and Kenneth Brown, both from ODU, for valuable inputs. Professor Anna Jeng, ODU, is also gratefully acknowledged for access to Malvern Zetasizer. The authors also thank the Reviewers of this manuscript for their valuable comments. 


\section{References}

1 N. Toshima and T. Yonezawa, New J. Chem., 1998, 22, 1179-1201.

2 R. Ferrando, J. Jellinek and R. L. Johnston, Chem. Rev., 2008, 108, 845-910.

3 H. Yasuda and Y. Yoshimura, Catal. Lett., 1997, 46, 43-48.

4 E. Antolini, Energy Environ. Sci., 2009, 2, 915-931.

5 J. Kua and W. A. Goddard, J. Am. Chem. Soc., 1999, 121, 10928-10941.

6 B. Lim and Y. Xia, Angew. Chem., Int. Ed., 2011, 50, 76-85.

7 A.-X. Yin, X.-Q. Min, Y.-W. Zhang and C.-H. Yan, J. Am. Chem. Soc., 2011, 133, 3816-3819.

8 Q. Yuan, Z. Zhou, J. Zhuang and X. Wang, Chem. Commun., 2010, 46, 1491-1493.

9 B. Lim, J. Wang, P. H. C. Camargo, C. M. Cobley, M. J. Kim and Y. Xia, Angew. Chem., Int. Ed., 2009, 48, 6304-6308.

10 B. Lim, M. Jiang, P. H. C. Camargo, E. C. Cho, J. Tao, X. Lu, Y. Zhu and Y. Xia, Science, 2009, 324, 1302-1305.

11 S. Guo, S. Dong and E. Wang, ACS Nano, 2010, 4, 547-555.

12 B. Lim, J. Wang, P. H. C. Camargo, M. Jiang, M. J. Kim and Y. Xia, Nano Lett., 2008, 8, 2535-2540.

13 X. Huang, H. Zhang, C. Guo, Z. Zhou and N. Zheng, Angew. Chem., Int. Ed., 2009, 48, 4808-4812.

14 S. E. Habas, H. Lee, V. Radmilovic, G. A. Somorjai and P. Yang, Nat. Mater., 2007, 6, 692-697.

15 S. Cheong, J. D. Watt and R. D. Tilley, Nanoscale, 2010, 2, 2045-2053.

16 A. R. Tao, S. Habas and P. Yang, Small, 2008, 4, 310-325.

17 M. Brust, M. Walker, D. Bethell, D. J. Schiffrin and R. Whyman, J. Chem. Soc., Chem. Commun., 1994, 801-802.

18 D. V. Leff, L. Brandt and J. R. Heath, Langmuir, 1996, 12, 4723-4730.

19 A. C. Templeton, W. P. Wuelfing and R. W. Murray, Acc. Chem. Res., 2000, 33, 27-36.

20 P. J. G. Goulet and R. B. Lennox, J. Am. Chem. Soc., 2010, 132, 9582-9584.

21 Y. Li, O. Zaluzhna, B. Xu, Y. Gao, J. M. Modest and Y. J. Tong, J. Am. Chem. Soc., 2011, 133, 2092-2095.

22 V. P. Sheela, W. Xiao, S. Han, X. Zhou, S. Albin and R. Balasubramanian, J. Mater. Chem., 2011, 21, 6395-6400.

23 K. B. Stavens, S. V. Pusztay, S. H. Zou, R. P. Andres and A. Wei, Langmuir, 1999, 15, 8337-8339.

24 Y. Sun, Y. Yao, C.-G. Yan, Y. Han and M. Shen, ACS Nano, 2010, 4, 2129-2141.

25 J. Alvarez, J. Liu, E. Roman and A. E. Kaifer, Chem. Commun., 2000, 1151-1152.

26 J. Yang, J. Y. Lee, T. C. Deivaraj and H. P. Too, Colloids Surf., A, 2004, 240, 131-134.

27 K. Wikander, C. Petit, K. Holmberg and M. P. Pileni, Langmuir, 2006, 22, 4863-4868.

28 L. S. Ott and R. G. Finke, Coord. Chem. Rev., 2007, 251, 1075-1100.
29 M. J. Hostetler, C. J. Zhong, B. K. H. Yen, J. Anderegg, S. M. Gross, N. D. Evans, M. Porter and R. W. Murray, J. Am. Chem. Soc., 1998, 120, 9396-9397.

30 J. A. Creighton and D. G. Eadon, J. Chem. Soc., Faraday Trans., 1991, 87, 3881-3891.

31 Analysis of multiple TEM images of the purified nanoparticles showed that a majority $(72.5 \%)$ of them were anisotropic.

32 M. J. Hostetler, J. E. Wingate, C.-J. Zhong, J. E. Harris, R. W. Vachet, M. R. Clark, J. D. Londono, S. J. Green, J. J. Stokes, G. D. Wignall, G. L. Glish, M. D. Porter, N. D. Evans and R. W. Murray, Langmuir, 1998, 14, 17-30.

33 S. Maksimuk, X. Teng and H. Yang, J. Phys. Chem. C, 2007, 111, 14312-14319.

34 R. Balasubramanian, Z. M. Kalaitzis and W. Cao, J. Mater. Chem., 2010, 20, 6539-6543.

35 N. Toshima, M. Harada, T. Yonezawa, K. Kushihashi and K. Asakura, J. Phys. Chem., 1991, 95, 7448-7453.

36 H. Ye and R. M. Crooks, J. Am. Chem. Soc., 2007, 129, 3627-3633.

37 Y. Liu, M. Chi, V. Mazumder, K. L. More, S. Soled, J. D. Henao and S. Sun, Chem. Mater., 2011, 23, 4199-4203.

38 B. Lim, M. J. Jiang, T. Yu, P. H. C. Camargo and Y. N. Xia, Nano Res., 2010, 3, 69-80.

39 J. Watt, N. Young, S. Haigh, A. Kirkland and R. D. Tilley, Adv. Mater., 2009, 21, 2288-2293.

40 H. Song, F. Kim, S. Connor, G. A. Somorjai and P. Yang, J. Phys. Chem. B, 2005, 109, 188-193.

41 A. W. Bosman, A. P. H. J. Schenning, R. A. J. Janssen and E. W. Meijer, Chem. Ber., 1997, 130, 725-728.

42 M. P. Pileni, J. Phys. Chem. C, 2007, 111, 9019-9038.

43 J. J. Chen and N. Winograd, Surf. Sci., 1995, 326, 285-300.

44 K. Naoe, C. Petit and M. P. Pileni, Langmuir, 2008, 24, 2792-2798.

45 F. Gao, Z. J. Li, Y. L. Wang, L. Burkholder and W. T. Tysoe, J. Phys. Chem. C, 2007, 111, 9981-9991.

46 X. Teng, M. Feygenson, Q. Wang, J. He, W. Du, A. I. Frenkel, W. Han and M. Aronson, Nano Lett., 2009, 9, 3177-3184.

47 N. Miyaura and A. Suzuki, Chem. Rev., 1995, 95, 2457-2483.

48 M. B. Thathagar, J. Beckers and G. Rothenberg, J. Am. Chem. Soc., 2002, 124, 11858-11859.

49 L. Strimbu, J. Liu and A. E. Kaifer, Langmuir, 2003, 19, 483-485.

50 J. Han, Y. Liu and R. Guo, J. Am. Chem. Soc., 2009, 131, 2060-2061.

51 R. Narayanan and M. A. El-Sayed, Langmuir, 2005, 21, 2027-2033.

52 With as-prepared PdPt bimetallic nanoparticles as catalysts, bases such as potassium carbonate, triethylamine and sodium acetate were screened and $\mathrm{K}_{2} \mathrm{CO}_{3}$ gave higher yields of biphenyl. 